\title{
0 impacto psicológico do câncer de mama
}

\author{
The psychological impact of breast cancer
}

\author{
Maria Fernanda de Matos Maluf ${ }^{1}$, Lincon Jo Mori ${ }^{2}$, Alfredo Carlos S. D. Barros ${ }^{3}$
}

\begin{abstract}
Resumo
Este estudo tem como objetivo promover o conhecimento do impacto psicológico produzido pelo câncer de mama através da revisão da literatura internacional. A literatura médica, costuma avaliar as alterações psicológicas por que passam as mulheres com câncer de mama, por meio de questionários e/ou inventários de qualidade de vida, aplicados em pacientes no pós-operatório. Estas pesquisas são ambíguas em suas conclusões, ora mencionando que quanto mais conservadora a cirurgia menor o impacto psicológico causado pelas alterações na imagem corporal ora dizendo que não há diferenças significativas entre os grupos, isto é, entre cirurgias radicais e conservadoras. Porém, estes estudos não avaliam o impacto psicológico que 0 câncer de mama desde 0 início de sua descoberta (auto-exame e posterior diagnóstico), o que seria de grande valia, pois as pacientes passam, durante todo este processo, por períodos de luto relevantes. Através do conhecimento e da compreensão dos processos psíquicos por que passam a mulher com câncer de mama, durante todas as fases do tratamento, torna-se possível o entendimento de sua dinâmica psíquica: seus medos, angústias e fantasias que podem interferir em uma melhor resposta ao tratamento. D esta forma torna-se imprescindível a formação de uma equipemultidisciplinar, composta por psicólogos e médicos, para que juntos proporcionem à mulher com câncer de mama, um atendimento humanitário, completo, promovendo assim o restabelecimento da saúde em seu sentido mais amplo: o indivíduo visto como um todo, ou seja, como um ser biopsicossocial, que se relaciona com o meio em que vive, mas que precisa estar em harmonia consigo próprio (psicológico e o biológico reintegrados).
\end{abstract}

Palavras-chave: N eoplasias mamárias; Psicologia; Saúde mental; Luto; Atitude frente a morte.

\footnotetext{
1,2,3 D epartamento de M astologia do H ospital das Clínicas da Universidade de São Paulo

${ }^{1}$ Psicóloga, Especialista em Sexualidade H umana e M estranda em Ginecologia e O bstetrícia pela FM U SP

2 D outorando em Ginecologia e O bstetrícia pela FM USP

${ }^{3}$ Livre D ocente pela FM USP

Endereço para correspondência: M aria Fernanda de M atos M aluf - R. Itacolomi, 601 cj. 66 - H igienópolis - CEP 01243-020 - São Paulo (SP).
} 


\begin{abstract}
This study aims to promote the knowledge of the psychological impact of breast cancer through a medical literature review. The medical literature usually evaluates the psychological changes in women with breast cancer using questionnaires and/or quality of life checklists which are applied in post surgery patients. These researches are ambiguous in their conclusions, mentioning, sometimes, that breast conservation surgeries have less psychological impact caused by the alterations in body image and others that there are not significant differences between the two groups, that is, radical and conservative surgeries. However, these studies do not estimate the psychological impact of breast cancer since it is uncovered (by the self-examination and the posterior diagnosis), which would be very useful, because the patients undergo throughout all this process, important mourning periods. Through the knowledge and comprehension of the psychological process that women with breast cancer are subjected to during all the phases of treatment, it is possible to understand her psychological dynamic: their fears, distress and fantasies which would interfere with a better response to the treatment. It was concluded that the action of a multidisciplinary health care team, compounded by psychologists and physicians, working together, is important to offer to women with breast cancer a thorough and humanitarian treatment. This can promote health restoration as a whole, whit the patient seen as an indivisible biopsychosocial being, who has a relation with their environment and demand to be in harmony with themselves (a reintegration of the psychological and the biological).
\end{abstract}

Key words: Breast neoplasms; Psychology; M ental health; M ourning; Attitude to death.

\section{INTRO DUÇÃO}

0 câncer de mama é a neoplasia maligna que mais atinge 0 sexo feminino e é a maior causa de mortes por este tipo de doença ${ }^{1}$, sendo responsável por cerca de $20 \%$ dos óbitos por câncer entre as mulheres².

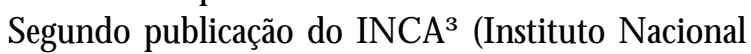
de (âncer), 0 câncer de mama continua a ser o mais incidente entre as mulheres, totalizando 53 casos a cada 100 mil pessoas, sendo que em 2003, esta estimativa era de 46 casos a cada 100 mil. $^{3,4} \mathrm{~A}$ estimativa de novos casos de câncer de mama, em 2005 é de 49.470, havendo 18.220 novos casos nas capitais brasileiras. ${ }^{4}$

O s dados consolidados de 2003 estimavam que, para cada 100 mil mulheres ocorressem 10.40 óbitos. Por conseguinte, a estimativa de óbitos foi de 9.335 casos no Brasil, correspondendo a 3.740 casos somente nas capitais.

A partir do momento em que a mulher descobre que tem um nódulo na mama, dá-se início a um processo interno de dúvidas e incertezas que podem ou não ser "amenizadas" através do exame físico e exames radiológicos.

$\mathrm{H}$ avendo a confirmação de que aquele achado é um tumor maligno, a mulher passará por várias fases de conflito interno que oscilam desde a negação da doença, onde a paciente (e familiares) procuram diversos profissionais na esperança de que algum deles lhe dê um diagnóstico contrário aos achados, até a fase final onde há a aceitação da existência do tumor. ${ }^{5}$

Esses conflitos são demonstrações das alterações psicológicas por que passam a mulher portadora de câncer de mama e seus familiares, e que não terminam com a cirurgia, mas que vão além, com os tratamentos como a quimioterapia, radioterapia e hormônioterapia.

Assim, vimos a importância da realização de um levantamento bibliográfico sobre as últimas produções que abordassem o tema do 'impacto psicológico gerado pelo câncer de mama', visando assim a melhor compreensão dos processos internos (psicológicos) por que passa a mulher portadora de câncer de mama.

\section{MÉTO DO}

Para esta revisão, foi utilizada uma pesquisa no M edline, entre 1980 e 2004, cruzando-se os unitermos "câncer de mama x aspectos psicológicos" além da pesquisa em livros da área de Ginecologia e Psicologia, que abordavam temas como câncer de mama, luto e morte, psicologia hospitalar, entre outros.

Os idiomas utilizados em ambas as consultas (M edline eliteratura da área de G inecologia e Psicologia) foram 0 inglês e o português.

\section{DISCUSSÃO}

Q uando uma mulher faz 0 auto-exame periódico das mamas e encontra um nódulo, inicia-se imediatamente 
um processo interno de dúvidas e incertezas relacionadas à possibilidade de aquele achado ser ou não um câncer. Afinal, a palavra câncer contém em si, ainda, um grande estigma: é sinônimo de morte. A palavra câncer é originária do latim cancer cancrí, caranguejo. ${ }^{7}$

Este estigma pode ser claramente observado no diaa-dia, quando vemos pessoas que não mencionam a palavra câncer por acharem que podem atrair a doença para si ou a chamam de "aquela doença", ou de "aquilo". 0 estigma também é percebido em relação ao doente de câncer: "coitado, está com câncer" ou "coitado, ele era uma boa pessoa e morreu de câncer...".

No que diz respeito ao doente de câncer, nota-se que as pessoas sempre o tratam como quem está para morrer brevemente. Q uerem saber "quanto tempo" a pessoa ainda tem de vida (assim como o próprio paciente) e algumas vezes se padecem da situação ao invés de apoiar o indivíduo e motivá-lo a seguir em frente, o que ajudaria, e muito, a diminuir a incidência de depressão. 0 doente de câncer, como a maioria dos doentes, percebe no olhar do outro que este tem pena de sua situação, pois estes sentimentos, por mais que se tente disfarçar, transparecem.

Assim, quando a mulher tem o diagnóstico de câncer de mama, a primeira reação é de desespero: "estou com câncer, vou morrer!". N este momento há uma real percepção da finitude da vida humana, através da antecipação da presença da morte. Este impacto inicial é o marco dos problemas psicológicos causados pelo tratamento do câncer de mama.

A reação ao diagnóstico de câncer de mama, depende das características de personalidade da paciente, da doença, das variáveis do tratamento, de sua interação com a doença e de fatores ambientais ${ }^{5}$.

A pós o choque, e depois de muitas vezes, relutar contra esta idéia, esta mulher vai a procura deum médico com a esperança, ainda que pequena, de que lhe seja dito que ela não tem nenhum tumor maligno. Então, ela é submetida a exames físicos e radiológicos (por exemplo, mamografia, ultra-sonografia das mamas e outros) para uma completa avaliação.

Frente ao não diagnóstico maligno, a sensação de alívio pela não morte é imensa. Porém, havendo a confirmação do câncer de mama, a mulher passa por várias fases de conflito interno que oscilam desde a negação da doença, onde a paciente choca-se com a notícia, não acreditando que tem câncer, afinal "estas coisas só acontecem com os outros e como foi acontecer comigo...". N esta fase, procura diversos profissionais na esperança de que algum deles Ihe dê um diagnóstico contrário aos achados, até a fase final de aceitação, onde há uma aceitação do diagnóstico de câncer de mama e a procura pelo tratamento. ${ }^{4}$

D urante estas etapas é muito importante que a paciente tenha um suporte por parte de seus familiares e amigos (suporte emocional). ${ }^{8}$ Porém, al gumas vezes isto se torna difícil devido aos fortes laços afetivos que unem estas pessoas e pelo desconhecimento da evolução e tratamento da doença. Isto é, se estas pessoas não conseguirem encarar a realidade da doença (o que é, suas características, tratamento, possibilidade de cura, entre outros) provavelmente não conseguirão dar apoio a esta mulher. Assim, o trabalho do psicólogo torna-se de grande valia tanto para a família quanto, e principalmente, para a paciente, que tem a oportunidade de expressar todas as suas angústias, medos (principalmente 0 da morte) e incertezas frente ao câncer, verbalizando conteúdos que possivelmente não contaria a um amigo e/ou familiar, com a diferença que estará tendo um suporte de um profissional preparado para isso e que poderá continuar a acompanhá-la durante todo este processo ${ }^{5,9,10}$.

Aliás, a maioria das pacientes não imagina que este tumor é uma doença sistêmica (em geral) e portanto a cirurgia muitas vezes não é o tratamento único (faz parte da terapêutica loco regional) e que será necessário um tratamento sistêmico, como quimioterapia e a hormonioterapia. Existe, também, uma grande confusão sobre os efeitos colaterais da quimioterapia e radioterapia, gerando mais ansiedade e medo - transtornos estes que podem e devem ser amenizados pelos especialista que acompanham o caso, como o mastologista, o oncologista, 0 radioterapêuta e o psicólogo.

Temos que nos ater ao fato de quea mulher portadora de câncer de mama passa por vários lutos ao longo do processo de tratamento: o primeiro pela existência da possibilidade de ter câncer, o segundo quando do diagnóstico, o terceiro quando do tratamento cirúrgico, um quarto luto gerado pela perda da imagem corporal e correlatos, um quinto luto causado pelas possíveis limitações que terá em conseqüência da cirurgia e um último causado pelos tratamentos quimoterápicos, radioterápicos e hormonioterápicos.

0 processo de luto é, por definição, um conjunto de reações diante de uma perda, envolvendo uma sucessão de quadros clínicos que se mesclam e se substituem. ${ }^{11}$ No sentido empregado por Freud, ${ }^{12}$ pode significar tanto 0 afeto da dor como sua manifestação externa.

$D$ e modo geral, o luto é a reação à perda de um ente querido, à perda de alguma abstração que ocupou 0 lugar e um ente querido, como o país, a liberdade ou 0 ideal de alguém, entre outros. ${ }^{11}$ 
$\mathrm{N}$ a realidade, o processo de luto pelo qual passa a mulher com câncer de mama é um momento em que esta tem a possibilidade de entrar em contato com seus conteúdos internos e os chocar com a nova realidade, elaborando isso, para que possa refazer psiquicamente sua auto-imagem, através do contato com uma nova realidade, neste caso, o câncer de mama. Porém, este processo é doloroso, sendo acompanhado desde uma tristeza atéuma profunda depressão, além de sentimentos como angústia, medo e desesperança. ${ }^{5}$ Assim é muito importante que a equipe médica que acompanha a paciente fique atenta aos sinais de problemas psicológicos demonstrados por esta, sabendo diferenciar a tristeza e a melancolia da depressão, que costuma acompanhar os pacientes com câncer, para que assim possam medicá-la, evitando assim futuras tentativas de suicídio. ${ }^{5,13}$

Q uando falamos em luto através do tratamento cirúrgico, queremos dizer que por um lado a cirurgia é "reconfortante" para a paciente, pois através do procedimento ela poderá "acabar com isso logo", ela será tratada, findando um "problema". Porém, a "alegria, o alívio" causado por esta primeira etapa do tratamento tem um certo tempo de duração, até o momento em que a paciente conscientiza-se cognitivo e emocionalmente e aí se inicia o luto. Luto pelo corpo perdido, pela feminilidade "roubada", sentimentos de menos valia por ser "menos mulher que as demais" (que possuem seios), entre outros. ${ }^{5,13}$

A literatura é rica em estudos sobre os aspectos psicológicos envolvidos no câncer de mama, principalmente após cirurgias radicais ou comparandose intervenções radicais a conservadoras. Porém, estes estudos medem o psicológico através de inventários de qualidade de vida e não através de uma inter-relação entre estes instrumentos e 0 acompanhamento psicológico adequado., 3,5,13-24

As avaliações sobre qualidade de vida abrangem questões sobre auto-estima, imagem corporal esexualidade (encontrada em alguns instrumentos), isto é, avaliam ou tentam avaliar fatores internos constituintes do self psicológico feminino. ${ }^{14} 0$ slf psicológico feminino é 0 eu feminino, é o ser e sentir-se como mulher: ser feminina, ter um corpo feminino, ter seios, menstruar e ser capaz de envolver-se em uma relação sexual ${ }^{15}$.

De modo geral, o câncer de mama tem como características, no self feminino, um aumento no senso de responsabilidade em relação a si mesma, uma maior vulnerabilidade, mudanças na auto-estima, raiva, medo da morte, de mudanças e da perda e mudanças na autoimagem, ${ }^{14}$ perda da feminilidade. ${ }^{15}$

Schain et al ${ }^{16}$ (1994) em estudo com 142 pacientes submetidas a mastectomia $(n=66)$ e a lumpectomia com irradiação $(n=76)$ demonstram que reações psicológicas mais importantes ocorridas nas pacientes com câncer de mama referem-se ao tipo de cirurgia realizada. D estas, as que foram submetidas à mastectomia apresentam sentimentos negativos persistentes e mais intensos a respeito de seu corpo do que àquelas submetidas a cirurgias conservadoras.

De modo geral, os estudos mostram que pacientes submetidas a cirurgias conservadoras como, por exemplo, a quadrantectomia e a lumpectomia (ou tumorectomia) apresentam uma menor comorbidade psicológica (por exemplo, depressão) do que as pacientes submetidas a cirurgias radicais. ${ }^{17-19} \mathrm{H}$ á algumas evidências de que a este tipo de cirurgia ofereça uma "proteção psicológica" contra possíveis distúrbios psicológicos, principalmente em pacientes joven $\mathrm{s}^{20} 0$ que não é observado em pacientes mais velhas. ${ }^{13}$ Isto pode ser observado em estudo conduzido por D orval et al, ${ }^{20}$ (1998) com 124 pacientes, 47 submetidas à mastectomia parcial e 77 a mastectomia radical, no qual observou-se que mais da metade das pacientes com idade inferior a 50 anos submetidas à mastectomia radical apresentavam um alto nível de sofrimento em todos os períodos do estudo ( 3 meses, 18 meses e 8 anos), quando comparadas às mulheres de mesma faixa etária do grupo submetido à mastectomia parcial.

Porém, outros estudos mostram não haver benefícios psicológicos entre este dois tipos de cirurgia, exceto em pacientes submetidas à mastectomia entre os 25 e os 47 anos, onde é possível notar escores mais baixos com relação a auto-imagem, quando comparadas àquelas submetidas a cirurgias conservadoras. ${ }^{21,22} \mathrm{Q}$ uanto à mastectomia, as pacientes verbalizam mais sentimentos depressivos e pensamentos transitórios sobre suicídio $(40 \%)$ durante 0 período entre 0 tratamento e das entrevistas, do que as pacientes submetidas à lumpectomia (7\%), em estudo conduzido por $\mathrm{M}$ argolis et al. ${ }^{23}$ (1990), devido a alteração visível da auto-imagem destas pacientes em comparação às pacientes submetidas à cirurgias conservadoras.

Em relação à reconstrução mamária imediata, os estudos mostram que este tipo de intervenção possui vantagens relacionadas a um melhor resultado estético e custo benefício para mulheres que a realizaram imediatamente após a cirurgia. Sessenta e oito por cento das pacientes submetidas à reconstrução imediata disseram-se muito satisfeitas com o resultado estético da cirurgia, em estudo realizado por Al-G hazal et al. ${ }^{18}$ Quando se compara o impacto psicológico da reconstrução imediata com grupos de reconstrução 
tardia constata-se, neste último grupo, um grande nível de sofrimento psíquico e rebaixamento das funções psíquicas aliados a uma baixa auto-imagem. 24,26

0 tratamento quimioterápico, seja pré e, principalmente, pós-operatório, provoca reações de luto pelo impacto nas mudanças corpóreas como a queda de cabelo, muitas vezes expressa como maior preocupação (no íntimo espelha o que a alopécia representa na psique da paciente: a diminuição da feminilidade, o estigma do câncer, o estigma da morte, a rejeição do sentimento de piedade, o preconceito, autodefesa, uma fase de rejeição). 0 utras mudanças corpóreas e não fisiológicas incluem, mal-estar geral (inclusive na possibilidade de diminuição da imunidade e infecções oportunistas), náuseas e vômitos importantes, diminuição da função cognitiva (como alterações na memória entre outras), quadro clínico decorrente de hipoestrogeinismo por diminuição da função ovariana (principalmente em pacientes pré-menopausada, podendo desenvolver diminuição da lubrificação vaginal, atrofia vaginal, fogachos, amenorréia). 2,10,14, 16

$\mathrm{N}$ esta fase, 0 apoio familiar e o suporte médicopsicológico pode amenizar os possíveis efeitos colaterais com medicações específicas e sempre orientando a paciente e os familiares das possíveis evoluções, diminuindo assim a tensão e o medo do incerto.

Um grande número de estudos focados no ajustamento psicológico, compara pacientes mastectomizadas com outras submetidas a cirurgias conservadoras, chegando a conclusões de que quanto mais conservadora a cirurgia, menor o impacto psicológico causado pela perda da auto-imagem. Porém, não há unanimidade quanto a isso, havendo outros estudos que afirmam não haver diferença entre 0 impacto psicológico em pacientes submetidas a mastectomia radical ou cirurgia conservadora. ${ }^{5}$

As alterações psicológicas que acompanham 0 diagnóstico e tratamento do câncer de mama iniciam-se a partir do momento que a mulher suspeita de que o nódulo que descobriu, através do auto-exame, possa ser um câncer.

O s estudos comparativos entre cirurgias conservadoras e radicais mostram e concluem, de modo geral, que a opção entre estes tipos de intervenção depende e muito, do entendimento que a paciente faz do que seria um tratamento mais completo, ou seja, pacientes preferem a mastectomia radical por sua "maior eficácia" no tratamento do câncer de mama e também por não requererem radioterapia. ${ }^{14}$

É de grande relevância que todas as pacientes diagnosticadas com câncer de mama tenham um adequado suporte psicológico durante todas as fases do tratamento pois, como referido no estudo de M oorey et al. ${ }^{25}$ (1989), entre 25 e 35\% das mulheres com câncer de mama irão desenvolver ansiedade e/ou depressão em algum estágio do tratamento e para muitas isso é incessante sem ajuda.

$D$ evemos nos ater que o adoecer é uma experiência única, uma experiência de desordem, que adquire um sentido específico no momento existencial dessa mulher, com significados que os sintomas, as experiências com 0 tratamento e as relações interpessoais passam a ter no contexto de sua vida.

D esta forma, esperamos que esta revisão tenha plantado uma semente sobre a importância de uma adequada intervenção psicológica durante o diagnóstico e tratamento do câncer de mama, agindo em prol de um bom prognóstico.

\section{REFERÊN CIAS}

1. H albeH W. Tratado deginecologia. 3aed. São Paulo: Roca; 2000. vol. 3.

2. Frost $M H$, Suman $V J$, RummansTA, D oseAM, Taylor $M$, N ovotny P, et al. Physical, psychological and social wellbeing of women with breast cancer: theinfluence of disease phase. Psychooncology. 2000;9(3):221-31.

3. Instituto N acional de Câncer; M inistério da Saúde. Estimativas deincidênciaemortalidade por câncer demama no Brasil, 2003 [monografia na Internet]. Rio deJ aneiro (Brasil): IN CA; 2003 [citado em 20 de maio de 2003]. D isponível em: http://www.inca.gov.br

4. Instituto N acional de Câncer; M inistério da Saúde. Estimativas deincidênciaemortalidadepor câncer demama no Brasil, 2005 [monografia na Internet]. Rio de] aneiro (Brasil): IN CA; 2004 [citado em 16 dedezembro de2004]. D isponível em: http://www.inca.gov.br

5. M aluf M FM . A sexualidade das pacientes submetidas a mastectomia radical [monografia]. São Paulo: Universidade deSão Paulo; 2004.

6. Backus VP. Psychiatric aspects of breast cancer. $\mathrm{H}$ arv Rev Psychiatry. 2002;10(5):307-14.

7. CunhaAG. Dicionário etimológico dalínguaportuguesa. $2 a$ ed. rev. ampl. Rio deJaneiro: N ova Fronteira; 1998. p. 146.

8. Rodrigues DP, M elo EM, Silva RM, M amede M V. 0 suporte social para atender as necessidades de mulheres mastectomizadas. Rev Bras C ancerol. 1998;44(3):231-8.

9. CamposT CP. Psicologia hospitalar: a atuação do psicólogo em hospitais. São Paulo: EPU ; 1995.

10. Kaplan H S. A neglected issue: the sexual side effects of current treatments for breast cancer. J Sex M arital Ther. 1992;18(1):3-19.

11. Parkers CM . Luto: estudo sobre a perda na idade adulta. Tradução deM aria H elena Franco Bromberg. São Paulo: Summus; 1998. 
12. Freud S. Luto emelancolia. Rio de J aneiro: I mago; 1976. Edição standard obras psicológicas completas, vol. 3.

13. Fallowfield LJ, H all A. Psychological and sexual impact of diagnosis and treatment of breast cancer. $\mathrm{Br} \mathrm{M}$ ed Bull. 1991;47(2):388-99.

14. Wilmoth M C, RossJA. Women'sperception: breast cancer treatment and sexuality. C ancer Pract. 1997;5(6):353-9.

15. W ilmoth M C. Theaftermath of breast cancer: an altered sexual self. Cancer N urs. 2001;24(4):278-86.

16. Schain WS, D 'Angelo T M , D unn M E, Lichter AS, Pierce $L J$. M astectomy versus conservative surgery and radiation therapy: psychosocial consequences. Cancer. 1994;73(4):1221-8.

17. Cohen L, H ack T F, M oor C, KatzJ, G ossPE. The effects of type of surgery and time on psychological adjustment in woman after breast cancer treatment. Ann Surg 0 ncol. 2000;7(6):427-34.

18. Al-G hazal SK, Fallowfield L, Blamey RW. Comparison of psychological aspects and patient satisfaction following breast conserving surgery, simple mastectomy and breast reconstruction. Eur J C ancer. 2000;36(15):1938-43.

19. Taylor SE, Lichtman RR, Wood JV, Bluming AZ, D osik GM , Leibowitz RL. I IIness related and treatment related factorsin psychological adjustment to breast cancer. $C$ ancer. 1985;55(10):2506-13.
20. O rval M, M aunsell E, D eschenes L, Bris-son J. Type of mastectomy and quality of lifefor long term breast carcinoma survivors. Cancer. 1998;83(10):2130-8.

21. Poulsen B, Graversen H P, Beckmann J, Blichert-Toft M . A comparative study of post-operativepsychosocial function in women with primary operablebreast cancer randomized to breast conservation therapy or mastectomy. Eur J Surg O ncol. 1997;23(4):327-34.

22. Ganz PA, Schag AC, Polinsky M L, Tan SJ. Breast conservation versus mastectomy. Is there a difference in psychological adjustment or quality of lifein theyear after surgery?C ancer. 1992;69(7):1729-38.

23. M argolis G, Goodman RL, Rubin A. Psychological effects of breast-conserving cancer treatment and mastectomy. Psychosomatics. 1990;31(1):33-9.

24. Arcourt D M , Rumsey N J, Ambler N R, C awthirn SJ, Reid $C D, M$ addox PR, et al. The psychological effect of mastectomy with or without breast reconstruction: a prospective, multicenter study. Plast Reconstr Surg. 2003;111(3):1060-8.

25.0 orey $\mathrm{S}, \mathrm{G}$ reer $\mathrm{S}$. Psychological therapy for patients with cancer: a new approach. O xford: H einemann; 1989.

26. M aguireP. Lateadversepsychological sequel of breast cancer and itstreatment. Eur J Surg O ncol. 1999;25(3):317-20. 